\title{
Profiling of miRNAs in pediatric asthma: Upregulation of miRNA-221 and miRNA-485-3p
}

\author{
FENG LIU $^{1 *}$, HOU-BING QIN ${ }^{1 *}$, BING XU ${ }^{2}$, HUI ZHOU $^{3}$ and DE-YU ZHAO ${ }^{1}$ \\ ${ }^{1}$ Department of Respiratory Medicine, Nanjing Children's Hospital Affiliated to Nanjing Medical University, Nanjing 210029; \\ ${ }^{2}$ Department of Emergency, Shanghai Jiaotong University Affiliated Sixth People's Hospital, Shanghai 200233; \\ ${ }^{3}$ Nanjing Maternity and Child Health Hospital of Nanjing Medical University, Nanjing 210004, P.R. China
}

Received March 23, 2012; Accepted August 6, 2012

DOI: $10.3892 / \mathrm{mmr} .2012 .1030$

\begin{abstract}
The aim of this study was to investigate the expression profiles of microRNAs (miRNAs) in pediatric asthma and to determine candidate miRNAs responsible for the pathogenesis of this disease. Microarrays were used to detect the differences in the miRNA expression levels between asthmatic children and controls. Airway inflammation was evaluated by cell counting and tissue biopsy in an ovalbumin (OVA)-induced murine asthma model. Real-time polymerase chain reaction (PCR) was used to verify the differentially expressed miRNAs. The targets of the identified miRNAs were analyzed by bioinformatic analysis. The sprouty-related protein with an EVH1 domain-2 (Spred-2) protein content was assessed by western blotting. Differences were observed in the expression of miRNAs between the asthmatic children and controls. Upregulation of miRNA-221 and miRNA-485-3p in pediatric asthmatics and murine asthma models were verified by real-time PCR. Spred-2, a predicted target of miRNA-221 and miRNA-485-3p, was downregulated in murine asthma models. Upregulation of miRNA-221 and miRNA-485-3p may regulate the pathogenesis of asthma.
\end{abstract}

\section{Introduction}

Asthma is a one of the most common pediatric diseases and is characterized by eosinophilic airway inflammation, reversible airway obstruction, hyperresponsiveness and airway remodeling $(1,2)$. The prevalence of asthma is increasing in most countries $(3,4)$. Asthma causes substantial social impact and costs to public and private healthcare systems $(3,4)$. Pediatric asthma is different from adult asthma in terms of severity, natural history, response to treatment and mechanisms $(5,6)$.

Correspondence to: Dr De-Yu Zhao, Department of Respiratory Medicine, Nanjing Children's Hospital Affiliated to Nanjing Medical University, Nanjing 210029, P.R. China

E-mail: zhaodeyu98@126.com

"Contributed equally

Key words: miRNA, pediatric asthma, pathogenesis
Early diagnosis of pediatric asthma, although challenging, is highly important for effective treatment. To date, the available treatment modalities have not been sufficient to satisfactorily control asthma in children (7). Therefore, a new method for treating pediatric asthma is urgently required.

microRNAs (miRNAs) are a class of small, noncoding, single-stranded RNAs that regulate gene expression by binding to their target mRNA and triggering either repression of protein translation or RNA degradation $(8,9)$. miRNAs regulate development, differentiation, stem-cell differentiation, growth control, apoptosis and immune functions $(8,9)$. Several miRNAs are involved in the pathogenesis of asthma $(10,11)$.

An miRNA array analysis in murine models of acute and chronic asthma revealed that miRNA expression in the lungs changed following exposure to allergens, and suggested that several miRNAs may regulate the biological processes during the course of asthma development (12). However, these findings were in contrast to those of an miRNA array analysis in asthmatic adults in another study, which had shown that changes in miRNA expression do not appear to be involved in the development of the asthmatic phenotype or the anti-inflammatory action of the corticosteroid budesonide in adult asthmatic patients (13). To date, very little is known about miRNA expression profiles in pediatric asthma.

In the present study, miRNA array analysis was performed to determine miRNA expression profiles in asthmatic children. Sprouty-related EVH1 domain-containing protein (Spred) negatively regulates allergen-induced airway inflammation and hyperresponsiveness in murine asthma models by modulating interleukin (IL)-5 signaling (14). Among the upregulated miRNAs in pediatric asthma, miRNA-221 and miRNA-485-3p were predicted to bind to Spred-2 mRNA by bioinformatic analysis. Upregulation of miRNA-221 and miRNA-485-3p among pediatric asthmatics and murine asthma models were verified by real-time PCR. Spred-2 protein level was downregulated in murine asthma models.

\section{Materials and methods}

Study population. The study population consisted of 12 children (age, 4-6 years) admitted to Nanjing Children's Hospital, China. Six of these children were diagnosed as having allergic asthma. The other 6 were considered as the control group. 
Asthma was diagnosed on the basis of the recommendations of the Global Initiative for Asthma (GINA); according to GINA, pediatric patients are defined as having asthma if they have visited the hospital within the past 12 months due to wheezing without evidence of common cold, and if their forced expiratory volume in 1 second (FEV1) after inhalation of a $\beta_{2}$ agonist increased by $12 \%$ compared to prior to the inhalation. The results of the skin-prick tests were positive for all the asthmatic children. The children were first diagnosed as having allergic asthma and did not undergo any treatment. Venous blood samples were obtained from all the children. The study was approved by the Medical Ethics Committee of Nanjing Children's Hospital. Written consent was obtained from all parents.

miRNA microarray assay. Lymphocytes were collected from the blood by using a lymphocyte separation medium. Total RNA was isolated using TRIzol (Invitrogen, Carlsbad, CA, USA) and an miRNeasy Mini kit (Qiagen, Hilden, Germany). The sixth generation of miRCURY ${ }^{\mathrm{TM}}$ LNA Array (v.16.0) (Exiqon) contains more than 1,891 capture probes, covering all human, mouse and rat miRNAs annotated in miRBase 16.0 , as well as all viral miRNAs associated with these species. In addition, this array contains capture probes for 66 new miRPlus ${ }^{\mathrm{TM}}$ human miRNAs.

Ovalbumin (OVA)-induced murine asthma models. The BALB/c mice were randomly distributed into two groups $(n=6)$. The mice were kept for one week prior to the experiment. On day 0 and day 14, mice were sensitized with $20 \mu \mathrm{g}$ of OVA and $20 \mathrm{mg} \mathrm{Al}(\mathrm{OH})_{3}$ in $0.2 \mathrm{ml}$ PBS. Following sensitization, mice were exposed to either aerosolized 1\% OVA/PBS or PBS only for $20 \mathrm{~min}$ once a day on days 27, 28, 29 and 30 . On day 31 , mice were analyzed for cell numbers in the bronchoalveolar lavage fluid (BALF) and histological study.

Histological study. Lungs were removed from mice, fixed in $10 \%$ formalin for $24 \mathrm{~h}$, dehydrated, mounted in paraffin, sectioned to a thickness of $4 \mu \mathrm{m}$, and stained with hematoxylin and eosin (HE).

Real-time polymerase chain reaction (PCR). Real-time PCR was performed as described previously (15). Briefly, the miRNA first-strand synthesis kit was used to perform the first-strand synthesis. SYBR-Green PCR was then performed. U6 spliceosomal RNA (snRNA) was used as the endogenous control.

Bioinformatic analysis of miRNAs. Bioinformatic analysis of miRNAs was performed by a method described in the literature $(12,13)$. miRNA targets were analyzed using the public database TargetScan 6.0 (http://www.targetscan.org).

Western blotting. The removed lung was lysed in protein lysis buffer (50 mM Tris, $150 \mathrm{mM} \mathrm{NaCl}, 10 \mathrm{mM}$ EDTA, 1\% Triton X-100, $200 \mathrm{mM}$ sodium fluoride and $4 \mathrm{mM}$ sodium orthovanadate-containing protease inhibitors; $\mathrm{pH}$ 7.5). Protein concentration was measured by the Bradford method. Proteins were separated by $10 \%$ sodium dodecyl sulfate-polyacrylamide electrophoresis (SDS-PAGE) and transferred to nitrocellulose membranes

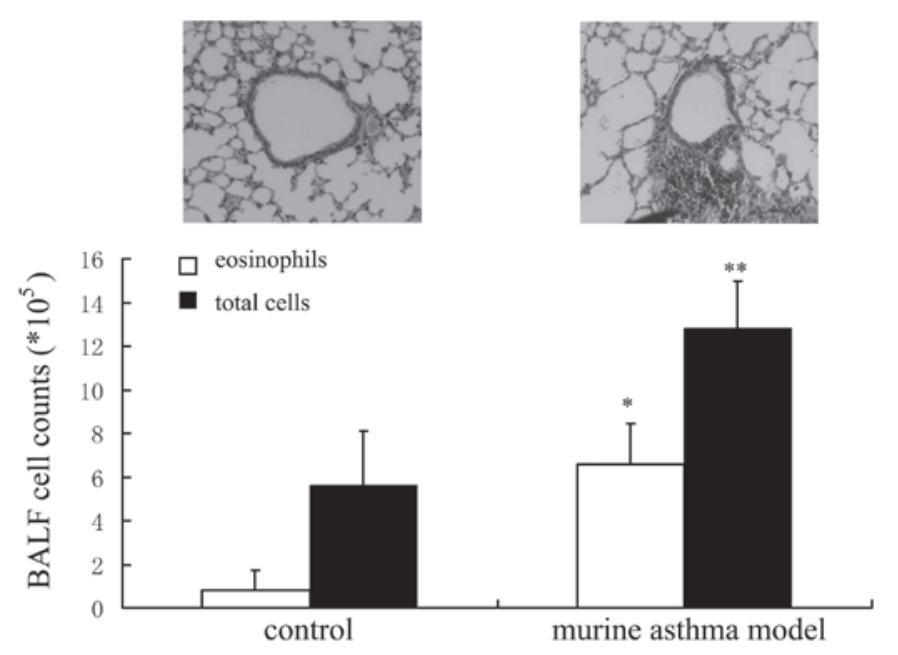

Figure 1. OVA-induced murine asthma models. The total cell and eosinophil counts were higher in OVA-induced murine asthma models compared with controls $\left(n=6,{ }^{*}\right.$ compared to the control eosinophils, $\mathrm{p}<0.05 ;{ }^{* *}$ compared to the control total cells, $\mathrm{p}<0.05$ ). The infiltration of leukocytes to lung tissue was observed in OVA-induced murine asthma models. OVA, ovalbumin; BALF, bronchoalveolar lavage fluid.

Membranes were blocked with $5 \%$ bovine serum albumin (BSA) in TBST (50 mM Tris, pH 7.5, $150 \mathrm{mM} \mathrm{NaCl,} \mathrm{0.05 \%}$ Tween-20). The membrane was incubated in 5\% BSA in TBST containing Spred-2 antibody (1:1000). Membranes were then washed extensively with TBST and then incubated with an appropriate secondary horseradish peroxidaselabeled antibody (Santa Cruz Biotechnology, Santa Cruz, CA, USA) at a 1:4,000 dilution. Bands were visualized by enhanced chemiluminescence (Amersham Biosciences, Piscataway, NJ, USA).

Statistical analysis. Statistical analysis was performed using the t-test. Differences between the groups were considered statistically significant when the p-value was $<0.05$.

\section{Results}

Differences in miRNA expression among the asthmatic and control-group children. The 6 asthmatic children had visited the hospital in the past 12 months due to wheezing without evidence of common cold, and their FEV1 values after inhalation of the $\beta 2$ agonist were $12 \%$ more than those preinhalation. The results of skin-prick tests were positive for all of the asthmatic children. The control-group children did not have a history of wheezing or chest tightness.

The microarray was used to detect the differences in the miRNA expression levels between these 2 groups. The expression levels of 36 miRNAs were significantly higher (more than two-fold) in the asthmatic children $(n=6)$ than in the controlgroup children $(\mathrm{p}<0.05)$. In addition, the expression levels of 47 miRNAs were significantly lower (more than two-fold) in the asthmatic children than in the control-group children $(\mathrm{p}<0.05$; Table I).

OVA-induced murine asthma models. The inflammation of airways and the infiltration of leukocytes to lung tissue are the 
Table I. Differences in miRNA expression among the asthmatic and control-group children.

\begin{tabular}{|c|c|c|c|c|c|}
\hline \multicolumn{2}{|c|}{ Upregulation } & \multicolumn{4}{|c|}{ Downregulation } \\
\hline miRNA & Fold & miRNA & Fold & miRNA & Fold \\
\hline hsa-miR-22 & 2 & hsa-miR-126 & 0.43 & hsa-let-7g & 0.48 \\
\hline hsa-miR-106b & 2.2 & hsa-miR-140-5p & 0.12 & hsa-miR-3614-3p & 0.17 \\
\hline hsa-miR-320a & 2.4 & hsa-let-7i & 0.47 & hsa-miR-23b & 0.38 \\
\hline hsa-miR-615-3p & 2.4 & hsa-miR-142-3p & 0.27 & hsa-miR-3926 & 0.19 \\
\hline hsa-miR-891a & 2 & hsa-miR-148a & 0.28 & hsa-miR-20a & 0.14 \\
\hline hsa-miR-877 & 2.7 & hsa-miR-182 & 0.37 & hsa-miR-33a & 0.2 \\
\hline hsa-miR-937 & 4.4 & hsa-miR-193a-3p & 0.44 & hsa-let-7d & 0.46 \\
\hline hsa-miR-196a & 3 & hsa-miR-29b & 0.29 & hsa-miR-30a & 0.36 \\
\hline hsa-miR-492 & 2.9 & hsa-miR-3607-5p & 0.23 & hsa-let-7a & 0.23 \\
\hline hsa-miR-485-3p & 2.2 & hsa-miR-335 & 0.11 & hsa-miR-27b & 0.22 \\
\hline hsa-miR-640 & 2.9 & hsa-miR-98 & 0.23 & hsa-miR-4284 & 0.13 \\
\hline hsa-miR-675 & 2.6 & hsa-miR-96 & 0.45 & & \\
\hline hsa-miR-554 & 5.4 & hsa-miR-195 & 0.1 & & \\
\hline hsa-let-7b & 2.7 & hsa-miR-143 & 0.37 & & \\
\hline hsa-miR-551b & 2.9 & hsa-miR-660 & 0.47 & & \\
\hline hsa-miR-320c & 2.5 & hsa-miR-532-5p & 0.45 & & \\
\hline hsa-miR-513b & 3.2 & hsa-miR-192 & 0.33 & & \\
\hline hsa-miR-320d & 2.2 & hsa-miR-4301 & 0.12 & & \\
\hline hsa-miR-605 & 3.7 & hsa-miR-362-3p & 0.21 & & \\
\hline hsa-miR-523 & 2.2 & hsa-miR-15b & 0.45 & & \\
\hline hsa-miR-665 & 4.4 & hsa-miR-744 & 0.41 & & \\
\hline hsa-miR-1260 & 2.2 & hsa-miR-15a & 0.3 & & \\
\hline hsa-miR-3202 & 2.1 & hsa-miR-30e & 0.27 & & \\
\hline hsa-miR-224 & 5.6 & hsa-miR-7 & 0.2 & & \\
\hline hsa-miR-221 & 2.3 & hsa-miR-199a-5p & 0.43 & & \\
\hline hsa-miR-4288 & 2.1 & hsa-miR-374a & 0.33 & & \\
\hline hsa-miR-4300 & 2 & hsa-miR-125b & 0.32 & & \\
\hline hsa-miR-491-3p & 3.4 & hsa-miR-126 & 0.45 & & \\
\hline hsa-miR-4306 & 2 & hsa-miR-576-5p & 0.36 & & \\
\hline hsa-miR-4268 & 3.1 & hsa-miR-324-5p & 0.47 & & \\
\hline hsa-miR-3171 & 2.6 & hsa-miR-20b & 0.32 & & \\
\hline hsa-miR-1246 & 2.4 & hsa-miR-20a & 0.28 & & \\
\hline hsa-miR-620 & 2 & hsa-miR-451 & 0.16 & & \\
\hline hsa-miR-938 & 3 & hsa-miR-138-1 & 0.35 & & \\
\hline hsa-miR-1280 & 2.3 & hsa-miR-424 & 0.19 & & \\
\hline hsa-miR-483-3p & 4.9 & hsa-miRPlus-I874 & 0.41 & & \\
\hline
\end{tabular}

Microarray analysis was performed to detect the differences in miRNA expression levels. Thirty-six miRNAs were significantly upregulated and 47 significantly downregulated two-fold in the asthmatic group, compared to the respective miRNAs in the control group ( $<<0.05$ ). miRNA, microRNA.

two major features of asthma. The total cell and eosinophil counts were higher in OVA-induced murine asthma models compared with controls. The infiltration of leukocytes to lung tissue was observed in OVA-induced murine asthma models (Fig. 1).

Upregulation of miRNA-221 and miRNA-485-3p in pediatric asthmatics and murine asthma models. Real-time PCR was used to confirm the differential expression of miRNA-221 and miRNA-485-3p in pediatric asthmatics and murine asthma models. miRNA-221 was upregulated approximately two-fold in pediatric asthmatics and approximately three-fold in murine asthma models. miRNA-485-3p was upregulated approximately 2.5 -fold in pediatric asthmatics and murine asthma models (Fig. 2).

Spred-2 protein level was downregulated in murine asthma models. The miRNA-221 and miRNA-485-3p targets were 


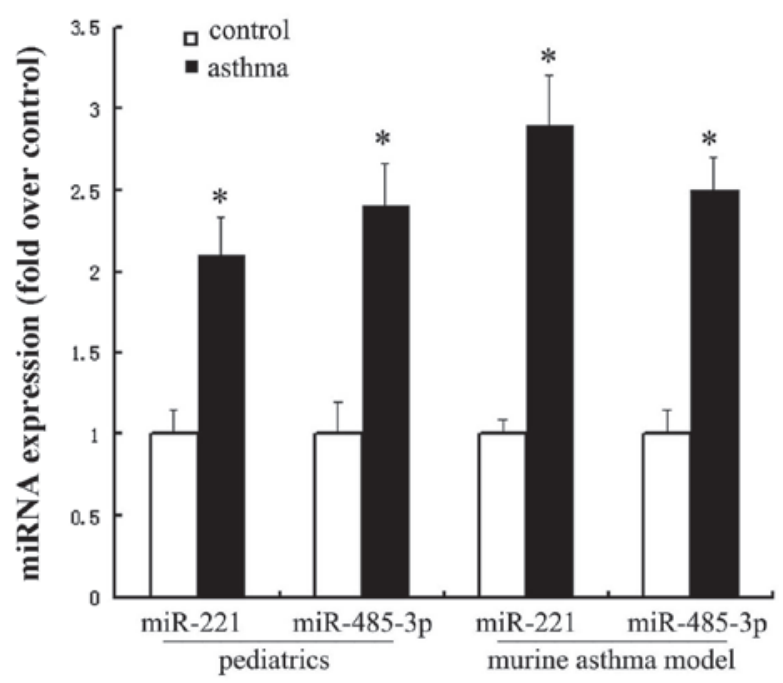

Figure 2. Upregulation of miRNA-221 and miRNA-485-3p in pediatric asthmatics and murine asthma models. miRNA-221 was upregulated approximately two-fold in pediatric asthmatics and approximately three-fold in murine asthma models. miRNA-485-3p was upregulated approximately 2.5-fold in pediatric asthmatics and murine asthma models $(n=6$, compared to the controls, $\mathrm{p}<0.05$ ). miRNA, microRNA.

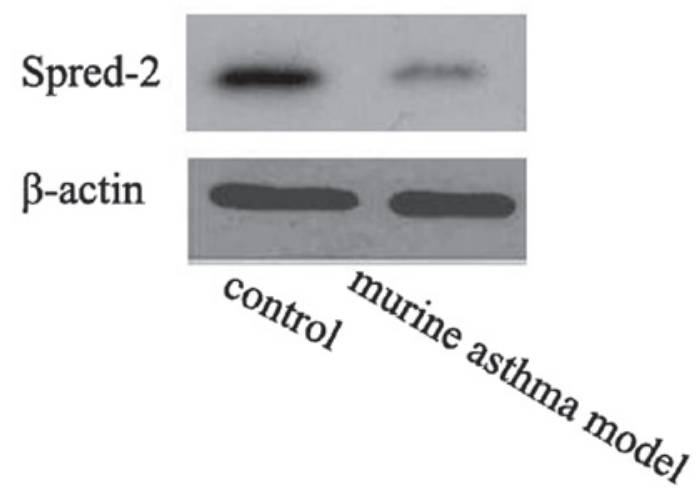

Figure 3. Spred-2 protein level was downregulated in murine asthma models. Spred-2 was the predicted target of miRNA-221 and miRNA-485-3p Representative immunoblots ( $n=3$ experiments) revealed that Spred- 2 protein level was downregulated in murine asthma models compared to the controls. miRNA, microRNA

analyzed using the public database TargetScan 6.0 (http:// www.targetscan.org). Spred-2 was the predicted target of miRNA-221 and miRNA-485-3p. The Spred-2 protein level was downregulated in murine asthma models compared to the controls (Fig. 3).

\section{Discussion}

In this study, 36 miRNAs were significantly upregulated and 47 significantly downregulated two-fold in the asthmatic group, compared to the respective miRNAs in the control group. A previous miRNA array analysis showed that miRNAs are not involved in the development of allergic asthma in adults (13). However, an miRNA array analysis in murine models of acute and chronic asthma revealed that miRNA expression in the lung changes on exposure to allergens and that several
miRNAs regulate biological processes during the course of asthma development (12).

Functional studies of miRNA in asthma also proved the critical role of miRNAs in asthma. Mice deficient in miRNA-155 show increased airway remodeling (10). Downregulation of miRNA-133a in a mouse model of allergic bronchial asthma has been shown to upregulate RhoA, resulting in increased airway contraction (11). In vivo inhibition of let-7 miRNAs inhibits the production of allergic cytokines and the development of the asthmatic phenotype (16). miRNA-26a is capable of regulating the hypertrophy of human airway smooth muscle cells by modulating the levels of glycogen synthase kinase-3 $\beta$ (17). Selective in vivo blockade of miRNA-126 suppresses the asthmatic phenotype (18). All these observations from previous studies prove that miRNAs play a critical role in the development of allergic asthma.

Spred-2 was downregulated in murine asthma models. Spred has been identified as a negative regulator of growth factor-mediated, Ras-dependent ERK activation (14). Spred negatively regulates allergen-induced airway inflammation and hyperresponsiveness in murine asthma models by modulating IL-5 signaling (14). Among the upregulated miRNAs in pediatric asthma, miRNA-221 and miRNA-485-3p were predicted to bind to Spred-2 mRNA by bioinformatic analysis.

Upregulation of miRNA-221 and miRNA-485-3p in pediatric asthmatics and murine asthma models were verified by real-time PCR. miRNA-221 favored mast cell adhesion and migration towards stem cell factor or antigen in Transwell migration assays, as well as cytokine production and degranulation in response to IgE-antigen complexes (19). miRNA-221 regulated cell cycle checkpoints in mast cells in response to acute activation stimuli (20). These results indicated that miRNA-221 may contribute to mast cell-related pathological conditions, such as asthma.

In conclusion, we applied miRNA microarray technique for screening the differential expression of miRNAs in asthmatic children. miRNA-221 and miRNA-485-3p were upregulated in pediatric asthmatics and murine asthma models. Spred-2, the predicted target of miRNA-221 and miRNA-485-3p, was downregulated in murine asthma models. miRNA-221 and miRNA-485-3p may regulate the pathogenesis of asthma.

\section{Acknowledgements}

This project was supported by grants (QYK09163, ZKX11012) from the Key Project supported by the Medical Science and Technology Development Foundation, Nanjing Department of Health.

\section{References}

1. Galli SJ, Tsai M and Piliponsky AM: The development of allergic inflammation. Nature 454: 445-454, 2008.

2. Tang ML and Powell CV: Childhood asthma as an allergic disease: rationale for the development of future treatment. Eur J Pediatr 160: 696-704, 2001.

3. Masoli M, Fabian D, Holt S and Beasley R: The global burden of asthma: executive summary of the GINA Dissemination Committee report. Allergy 59: 469-478, 2004.

4. Braman SS: The global burden of asthma. Chest 130: 4S-12S, 2006.

5. Phelan PD, Robertson CF and Olinsky A: The Melbourne Asthma Study: 1964-1999. J Allergy Clin Immunol 109: 189-194, 2002. 
6. Szefler SJ: Facing the challenges of childhood asthma: what changes are necessary? J Allergy Clin Immunol 115: 685-688, 2005.

7. MacDonald C, Sternberg A and Hunter PR: A systematic review and meta-analysis of interventions used to reduce exposure to house dust and their effect on the development and severity of asthma. Environ Health Perspect 115: 1691-1695, 2007.

8. Bartel DP: MicroRNAs: genomics, biogenesis, mechanism, and function. Cell 116: 281-297, 2004.

9. Ambros V: The functions of animal microRNAs. Nature 431: 350-355, 2004

10. Rodriguez A, Vigorito E, Clare S, et al: Requirement of bic/ microRNA-155 for normal immune function. Science 316 608-611, 2007.

11. Chiba Y and Misawa M: MicroRNAs and their therapeutic potential for human diseases: MiR-133a and bronchial smooth muscle hyperresponsiveness in asthma. J Pharmacol Sci 114: 264-268, 2010

12. Garbacki N, Di Valentin E, Huynh-Thu VA, et al: MicroRNAs profiling in murine models of acute and chronic asthma: a relationship with mRNAs targets. PLoS One 6: e16509, 2011.

13. Williams AE, Larner-Svensson H, Perry MM, et al: MicroRNA expression profiling in mild asthmatic human airways and effect of corticosteroid therapy. PLoS One 4: e5889, 2009.
14. Inoue H, Kato R, Fukuyama S, et al: Spred-1 negatively regulates allergen-induced airway eosinophilia and hyperresponsiveness. J Exp Med 201: 73-82, 2005.

15. Mayoral RJ, Pipkin ME, Pachkov M, van Nimwegen E, Rao A and Monticelli S: MicroRNA-221-222 regulate the cell cycle in mast cells. J Immunol 182: 433-445, 2009.

16. Polikepahad S, Knight JM, Naghavi AO, et al: Proinflammatory role for let-7 microRNAS in experimental asthma. J Biol Chem 285: 30139-30149, 2010.

17. Mohamed JS, Lopez MA and Boriek AM: Mechanical stretch up-regulates microRNA-26a and induces human airway smooth muscle hypertrophy by suppressing glycogen synthase kinase3beta. J Biol Chem 285: 29336-29347, 2010.

18. Mattes J, Collison A, Plank M, Phipps S and Foster PS: Antagonism of microRNA-126 suppresses the effector function of TH2 cells and the development of allergic airways disease. Proc Natl Acad Sci USA 106: 18704-18709, 2009.

19. Mayoral RJ, Deho L, Rusca N, et al: MiR-221 influences effector functions and actin cytoskeleton in mast cells. PLoS One 6: e26133, 2011.

20. Chun-Zhi Z, Lei H, An-Ling Z, et al: MicroRNA-221 and microRNA-222 regulate gastric carcinoma cell proliferation and radioresistance by targeting PTEN. BMC Cancer 10: 367, 2010. 